\title{
A Three-State Model for the Photophysics of Guanine
}

\author{
Luis Serrano-Andrés, ${ }^{* \dagger}$ Manuela Merchán, ${ }^{\dagger}$ Antonio Carlos Borin ${ }^{*}$ \\ Instituto de Ciencia Molecular, Universitat de València, Apartado 22085, ES-46071 \\ Valencia, Spain \\ and Instituto de Química, Universidade de São Paulo, Av. Prof. Lineu Prestes 748, \\ 05508-900, São Paulo, SP, Brazil
}

Supporting Information

*To whom correspondence should be addressed. E-mail: Luis.Serrano@uv.es.

${ }^{\dagger}$ Universitat de València.

¥ Universidade de São Paulo. 


\section{Additional computational details}

Most calculations reported in the present contribution have been performed using the CASPT2//CASSCF protocol, in which geometry optimizations, including minima, surface crossings, and minimum energy paths (MEPs) were carried at the multiconfigurational CASSCF level, whereas electronic energy computations use the second-order multiconfigurational perturbation approach, CASPT2. ${ }^{1,2,3,4}$ Different active spaces have been employed in order to ensure the converged character of the results. Initially, a $\pi$ active space of 12 electrons and 10 orbitals (the lowest nodeless $\pi$ orbital was kept inactive) was used to optimize $\pi \pi^{*}$-type states, whereas for $n \pi^{*}$ states the space was increased to 14 electrons and 12 orbitals, adding lone-pair in-plane orbitals. Additional extra-valence orbitals were included in the active space to assure the proper behavior of the computed paths, confirming the selection performed. Except when indicated, calculations used a double-zeta plus polarization $6-31 \mathrm{G}(\mathrm{d}, \mathrm{p})$ one-electron basis set in all atoms. Test calculations, mentioned in the text, were performed by using a larger ANO-S type basis set contracted to C,N,O [3s2p1d] / H [2s1p] to analyze the effect of increasing the quality of the basis set. The standard zeroth-order Hamiltonian was employed in the CASPT2 calculations, which include an imaginary level-shift correction of $0.2 \mathrm{au}$ in order to avoid the presence of intruder states. ${ }^{5}$ All calculations used the MOLCAS-6.0 set of programs, ${ }^{6}$ except for the computation of nonadiabatic coupling matrix elements (NACMEs) that were performed with the Gaussian-03 program. ${ }^{7}$

MEPs have been built as steepest descendent paths in a procedure ${ }^{8}$ which is based on a modification of the projected constrained optimization (PCO) algorithm of Anglada and Bofill ${ }^{9}$ and follows the Müller-Brown approach. ${ }^{10}$ Each step requires the minimization of the PEH on a hyperspherical cross section of the PEH centered on the initial geometry and characterized by a predefined radius. The optimized structure is taken as the center of a new hypersphere of the same radius, and the procedure is iterated until the bottom of the energy surface is reached. Mass-weighted coordinates are used, therefore the MEP coordinate corresponds to the so-called Intrinsic Reaction Coordinate (IRC), measured in $\mathrm{au}$, that is, bohr·amu ${ }^{1 / 2}$. To improve convergence different hypersphere radius were 
employed, as they are represented in the abscissa coordinate in the corresponding graphics. The full procedure is currently implemented in the MOLCAS-6.0 package ${ }^{6}$ and its technical description has been published elsewhere. ${ }^{8}$ At selected points of the MEP, CASPT2//CASSCF $(14,12) / 6-31 G(d, p)$ calculations including seven state-average roots were performed for the states of interest.

In this contribution conical intersection searches were carried out using the restricted Lagrange multipliers technique as included in the MOLCAS-6.0 package ${ }^{6}$ in which the lowest-energy point was obtained under the restriction of degeneracy between the two considered states. ${ }^{8}$ In principle, the crossings were obtained as minimum energy crossing points (MECPs), in which no non-adiabatic coupling matrix elements (NACMEs) were estimated. Minimum energy CIs (MECIs), that is, including NACMEs, were then recomputed to obtain the CIs ( $\mathrm{gs} / \pi \pi^{*} \mathrm{La}_{\text {a }}$ MECI. In all cases, the corresponding MECP and MECI structures were practically identical, with differences in bond lengths and angles smaller than $0.002 \AA$ and $2^{\circ}$, respectively. The description of the seam on Figure 6 was performed by combining in each of the points two constraints in the CASSCF optimization procedure: the degeneracy between the states and a fixed predefined distance from the origin geometry, i.e., the ( $\mathrm{gs} / \pi \pi^{*} \mathrm{La}$ ) $\mathrm{MECI}$ structure. The predefined distance is imposed as the radius of the hypersphere. That is, by increasing the radius and keeping the degeneracy condition the path of the seam connecting the two CIs can be obtained. With respect to the other gs/n $\pi^{*}$-type CIs, when the splitting of the CASPT2 states at the CASSCF geometries was too large, and in order to locate the conical intersection at a higher level of theory, CASPT2 calculations around the CASSCF optimized structures were performed in order to find the lowest-energy structure for the highest state which had the smallest energy difference between the computed states. The CASPT2 scans were performed by computing a grid of points along distortions involving the coordinates with the smallest values for the CASSCF gradients in the region of the crossing.

As a summary, in all the calculations of the present paper and at each optimized geometry, seven singlet states were computed using an active space of 14 electrons and 
12 active orbitals, $(14,12)$. Always, and in order to include the dynamic correlation effects to obtain the electronic energies, CASPT2 calculations were performed as described.

Regarding the molecular dynamics calculations represented in Figure 9 and discussed in the text, they contain two sample classical trajectories from a set of similar runs computed on the fly along the ${ }^{1}\left(\pi \pi^{*} \mathrm{~L}_{\mathrm{a}}\right)$ state PEHs starting close to the $\mathrm{FC}$ molecular geometry. They were obtained by using the velocity-Verlet algorithm ${ }^{11}$ with a constant time step of $1 \mathrm{fs}$ and employed ab initio two-state $\operatorname{SA}-\operatorname{CASSCF}(12,11) / 6-31 \mathrm{G}(\mathrm{d}, \mathrm{p})$ analytical gradients ${ }^{12}$ in the full space of coordinates, with control of the eventual loss of total energy in successive steps. Both CASSCF and CASPT2 energies were then used and they are represented in Figure 9. The two trajectories displayed start at the FC geometry, and they have been selected as representative of a group of ten to fifteen runs showing similar shapes and outcome, and obtained by initially distorting the molecule along the lowest-energy ground-state normal modes. The trajectories are part of a more complete and ongoing study on the photodynamics of the molecule in which different aspects of the methodology are being tested and implemented, for instance, the thorough inclusion of initial conditions by randomly sampling the vibrational modes of the resulting structures and adding different surface hopping algorithms, such as that initially implemented here based on surface selection by analysis of the configuration interaction vectors on successive simulation steps, known as the diabatic hop approach, ${ }^{13,14}$ or more sophisticated techniques like Tully's fewest switches procedure. ${ }^{15,16}$

Table SI1 compiles the Cartesian coordinates of the optimized minima and surface crossings obtained in the present contribution for the four guanine tautomers at the described CASSCF (or scanned CASPT2 for gs $/ \mathrm{n} \pi^{*}$-type CIs) level of theory, and includes also absolute energies at the CASSCF level of theory. Figure SI1 summarizes the IUPAC nomenclature for six-membered ring puckering distortions related to those occurring in DNA photochemistry, as mentioned in the paper. Figure SI2 displays the structure of several molecular orbitals of the $9 \mathrm{H}$-guanine molecule to be used in qualitative discussions. Figure SI3 displays a Linear Interpolation in Internal Coordinates 
(LIIC) profile initially employed to estimate the barrier for the $n_{O} \pi^{*} / \pi \pi^{*} L_{a}$ switch. The finally used $\left(\pi \pi^{*} L_{a} / n_{O} \pi^{*}\right)_{C I}$ was obtained by searching from the crossing obtained in the LIIC. Figure SI 4 includes the structure of the $\left(\mathrm{gs} / \pi \pi^{*} \mathrm{~L}_{\mathrm{a}}\right)_{\mathrm{MECI}}$ in the four molecules. Figure SI5 displays the structure of some important critical points characterized for guanine in the present paper. Figures SI6 to SI10 contain the MEP profiles computed from the FC region and along the ${ }^{1}\left(\pi \pi^{*} L_{a}\right)$ and ${ }^{1}\left(\pi \pi^{*} L_{b}\right)$ states for $7 \mathrm{H}$-guanine, $9 \mathrm{H}$ $6 \mathrm{OH}$-guanine, and $7 \mathrm{H}-6 \mathrm{OH}$-guanine, to be used in the discussions of the paper. Finally, Figure SI1 1 displays the structure of the $\left(\mathrm{gs} / \mathrm{n}_{\mathrm{N}} \pi^{*} \mathrm{~L}_{\mathrm{a}}\right)_{\mathrm{CI}}$ in $9 \mathrm{H}-6 \mathrm{OH}$-guanine.

Present Refs. 6 and 7 correspond to complete Refs. 57 and 60 and of the paper, respectively. 
Table S1. Cartesian coordinates $(\AA)$ of the CASSCF optimized structures obtained in the paper and absolute energies. 6-31G(d,p) basis set

\begin{tabular}{ccrc} 
Atom & $\mathrm{x}$ & $\mathrm{y}$ & $\mathrm{Z}$ \\
& \multicolumn{3}{c}{$9 \mathrm{H}$-guanine $(\mathrm{gs}) \min :-539.519315 \mathrm{au}$} \\
& & & \\
$\mathrm{C}$ & -2.249501 & -0.784664 & -0.094871 \\
$\mathrm{C}$ & -0.045112 & -0.683692 & 0.028750 \\
$\mathrm{C}$ & 0.034127 & 0.683348 & 0.015119 \\
$\mathrm{C}$ & -1.189236 & 1.451290 & -0.078768 \\
$\mathrm{C}$ & 2.029485 & -0.006620 & 0.131209 \\
$\mathrm{O}$ & -1.350561 & 2.640282 & -0.127447 \\
$\mathrm{~N}$ & -2.295962 & 0.583407 & -0.127188 \\
$\mathrm{~N}$ & -1.164417 & -1.469435 & -0.007377 \\
$\mathrm{~N}$ & 1.346052 & 1.094878 & 0.078927 \\
$\mathrm{~N}$ & 1.236650 & -1.125486 & 0.105290 \\
$\mathrm{~N}$ & -3.471138 & -1.416375 & -0.206585 \\
$\mathrm{H}$ & -3.170856 & 1.029541 & -0.294397 \\
$\mathrm{H}$ & -4.211325 & -1.013697 & 0.325984 \\
$\mathrm{H}$ & -3.395804 & -2.400279 & -0.069407 \\
$\mathrm{H}$ & 1.528232 & -2.074062 & 0.132866 \\
$\mathrm{H}$ & 3.096025 & -0.072016 & 0.187895
\end{tabular}

9H-guanine $\left(\text { no } \pi^{*}\right)_{\min }:-539.362033$ au

$\begin{array}{rrr}-2.253804 & -0.774120 & -0.088716 \\ -0.041741 & -0.678647 & 0.033884 \\ 0.040103 & 0.680891 & -0.066584 \\ -1.175293 & 1.428305 & -0.273773 \\ 2.034801 & -0.007407 & 0.099636 \\ -1.374139 & 2.622519 & 0.384586 \\ -2.320375 & 0.598169 & -0.206718 \\ -1.179541 & -1.457153 & 0.055624 \\ 1.352491 & 1.093022 & -0.027670 \\ 1.243774 & -1.120885 & 0.137799 \\ -3.486385 & -1.398256 & -0.174941 \\ -3.145430 & 0.947281 & -0.641262 \\ -4.194156 & -0.985401 & 0.394744 \\ -3.410004 & -2.379754 & -0.018791 \\ 1.534976 & -2.064896 & 0.231829 \\ 3.101392 & -0.067227 & 0.160348\end{array}$

9H-guanine $\left(\pi \pi^{*} \mathrm{Lb}\right)_{\min }:-539.307537 \mathrm{au}$

$\begin{array}{rrr}-2.308098 & -0.739415 & -0.198670 \\ -0.064043 & -0.739527 & 0.035755 \\ 0.030826 & 0.708902 & -0.009725 \\ -1.188077 & 1.440041 & -0.149566 \\ 2.054174 & -0.038499 & 0.283556 \\ -1.269207 & 2.674442 & -0.211089 \\ -2.303573 & 0.647454 & -0.204757 \\ -1.155651 & -1.432858 & -0.031010 \\ 1.310372 & 1.153232 & 0.090145\end{array}$


Table S1. Cartesian coordinates $(\AA)$ of the CASSCF optimized structures obtained in the paper. $6-31 \mathrm{G}(\mathrm{d}, \mathrm{p})$ basis set (cont).

\begin{tabular}{crrr} 
Atom & \multicolumn{1}{c}{ X } & y & \multicolumn{1}{c}{$\mathrm{Z}$} \\
$\mathrm{N}$ & 1.248743 & -1.183149 & 0.069396 \\
$\mathrm{~N}$ & -3.574951 & -1.326649 & -0.086828 \\
$\mathrm{H}$ & -3.180619 & 1.101706 & -0.333757 \\
$\mathrm{H}$ & -3.813809 & -1.546855 & 0.861765 \\
$\mathrm{H}$ & -3.629762 & -2.165050 & -0.627334 \\
$\mathrm{H}$ & 1.477404 & -2.053998 & 0.493139 \\
$\mathrm{H}$ & 3.092928 & -0.063358 & 0.018981
\end{tabular}

9H-guanine (gs/ $\left.\pi \pi^{*} \mathrm{La}\right) \mathrm{cl}:-539.347194$ au (gs)

$\begin{array}{lrrr}\mathrm{C} & -2.214908 & -0.665895 & -0.153890 \\ \mathrm{C} & -0.096231 & -0.850631 & -0.151980 \\ \mathrm{C} & -0.177063 & 0.510042 & 0.350654 \\ \mathrm{C} & -1.251314 & 1.379205 & -0.167511 \\ \mathrm{C} & 1.883220 & -0.030137 & 0.387204 \\ \mathrm{O} & -1.285414 & 2.578173 & -0.141222 \\ \mathrm{~N} & -2.293888 & 0.596928 & -0.718173 \\ \mathrm{~N} & -1.115479 & -1.466578 & -0.646935 \\ \mathrm{~N} & 1.096646 & 0.955869 & 0.667229 \\ \mathrm{~N} & 1.240608 & -1.141737 & -0.138554 \\ \mathrm{~N} & -3.349697 & -1.336014 & 0.211655 \\ \mathrm{H} & -3.163517 & 1.050586 & -0.893860 \\ \mathrm{H} & -3.964072 & -0.846742 & 0.825501 \\ \mathrm{H} & -3.190515 & -2.283670 & 0.474137 \\ \mathrm{H} & 1.664802 & -1.984653 & -0.444133 \\ \mathrm{H} & 2.943479 & -0.028332 & 0.539888\end{array}$

9H-guanine (gs/ $\pi \pi^{*}$ La)MECI: -539.350687 au (gs)

$\begin{array}{rrrr}\mathrm{N} & 0.205332 & 0.645311 & 1.970843 \\ \mathrm{~N} & 2.046343 & 0.193374 & 0.806739 \\ \mathrm{~N} & -0.381006 & 0.172052 & -1.998055 \\ \mathrm{~N} & -1.395351 & 0.555578 & 0.119657 \\ \mathrm{~N} & -1.693818 & -1.576770 & -1.010459 \\ \mathrm{C} & -0.220468 & 0.339788 & 0.702252 \\ \mathrm{C} & 0.944277 & 0.059534 & -0.008728 \\ \mathrm{C} & 1.572754 & 0.529130 & 1.946260 \\ \mathrm{C} & 0.929799 & 0.047396 & -1.485949 \\ \mathrm{C} & -1.372406 & -0.217396 & -1.105111 \\ \mathrm{O} & 1.867525 & -0.040822 & -2.208651 \\ \mathrm{H} & -0.482269 & 0.013469 & -2.975787 \\ \mathrm{H} & -1.041011 & -2.253694 & -1.342056 \\ \mathrm{H} & -2.251899 & -1.845654 & -0.232185 \\ \mathrm{H} & -0.351447 & 0.991074 & 2.716072 \\ \mathrm{H} & 2.160054 & 0.703903 & 2.825750 \\ & & & \end{array}$


Table S1. Cartesian coordinates $(\AA)$ of the CASSCF optimized structures obtained in the paper. 6-31G(d,p) basis set (cont).

9H-guanine (no $\left.\pi^{*}\right)_{\mathrm{Ts}}$ - $-539.371022 \mathrm{au}$

$\begin{array}{lrrr}\mathrm{N} & -2.322025 & 0.567604 & -0.150682 \\ \mathrm{~N} & -1.174364 & -1.476002 & 0.067471 \\ \mathrm{~N} & 1.379427 & 1.085942 & 0.051917 \\ \mathrm{~N} & 1.241358 & -1.137408 & 0.120907 \\ \mathrm{~N} & -3.457866 & -1.432685 & -0.199682 \\ \mathrm{C} & -2.246934 & -0.799622 & -0.072201 \\ \mathrm{C} & -0.042288 & -0.690482 & 0.079626 \\ \mathrm{C} & 0.036296 & 0.669441 & 0.038283 \\ \mathrm{C} & -1.192750 & 1.425405 & -0.089868 \\ \mathrm{C} & 2.045014 & -0.032181 & 0.113688 \\ \mathrm{O} & -1.421257 & 2.495845 & 0.749610 \\ \mathrm{H} & -3.151944 & 0.942104 & -0.551976 \\ \mathrm{H} & -4.216335 & -1.020667 & 0.298122 \\ \mathrm{H} & -3.384853 & -2.416765 & -0.064515 \\ \mathrm{H} & 1.526855 & -2.086345 & 0.177571 \\ \mathrm{H} & 3.108338 & -0.117740 & 0.031721\end{array}$

9H-guanine (gs/no $\left.\pi^{*}\right)_{\mathrm{cl}}$-539.373729 au (gs)

$\begin{array}{lrrr}\mathrm{N} & 1.483939 & 2.459040 & -0.336889 \\ \mathrm{~N} & -0.561673 & 1.568487 & 0.280685 \\ \mathrm{~N} & 2.208094 & -0.926681 & 0.331943 \\ \mathrm{~N} & 3.315871 & 0.980863 & -0.004833 \\ \mathrm{~N} & -0.409521 & 3.717021 & -0.655789 \\ \mathrm{C} & -0.135339 & -0.147196 & -0.247012 \\ \mathrm{C} & 1.316286 & 0.019738 & 0.001893 \\ \mathrm{C} & 1.991587 & 1.266604 & -0.209046 \\ \mathrm{C} & 0.109268 & 2.537282 & -0.235930 \\ \mathrm{C} & 3.391675 & -0.346344 & 0.314750 \\ \mathrm{O} & -0.504191 & -0.245283 & -1.401098 \\ \mathrm{H} & 4.061914 & 1.626721 & -0.040857 \\ \mathrm{H} & 4.311230 & -0.843013 & 0.527714 \\ \mathrm{H} & 0.196476 & 4.383949 & -1.056753 \\ \mathrm{H} & -1.382997 & 3.858462 & -0.719496 \\ \mathrm{H} & -1.549056 & 1.693494 & 0.357012\end{array}$


Table S1. Cartesian coordinates $(\AA)$ of the CASSCF optimized structures obtained in the paper. $6-31 \mathrm{G}(\mathrm{d}, \mathrm{p})$ basis set (cont).

9H-guanine $\left(\pi \pi^{*} \mathrm{La} / \text { no } \pi^{*}\right)_{\mathrm{CI}}:-539.347374 \mathrm{au}\left({ }^{1} \mathrm{La}\right)$

$\begin{array}{rrr}-2.304791 & 0.653086 & -0.184039 \\ -1.156168 & -1.452172 & -0.022280 \\ 1.356721 & 1.100195 & 0.009439 \\ 1.218598 & -1.141487 & 0.200660 \\ -3.577715 & -1.304608 & -0.045735 \\ -2.313175 & -0.754064 & -0.328098 \\ -0.088182 & -0.741343 & 0.041582 \\ 0.022259 & 0.702410 & -0.075395 \\ -1.148281 & 1.373851 & -0.186669 \\ 2.005370 & -0.000186 & 0.175352 \\ -1.306718 & 2.701649 & -0.248588 \\ -3.174855 & 1.121964 & -0.297282 \\ -3.662558 & -1.561318 & 0.920738 \\ -3.739556 & -2.122592 & -0.594384 \\ 1.529580 & -2.074436 & 0.326693 \\ 3.066142 & -0.064507 & 0.308003\end{array}$

9H-guanine $\left(\pi \pi^{*} \mathrm{Lb} / \mathrm{no} \pi^{*}\right)_{\mathrm{Cr}}:-539.305939$ au $\left(\mathrm{n} \pi^{*}\right)$

$\begin{array}{rr}-2.305796 & 0.649010 \\ -1.152403 & -1.433835 \\ 1.302233 & 1.156015 \\ 1.251328 & -1.188481 \\ -3.574622 & -1.325976 \\ -2.308538 & -0.737280 \\ -0.062058 & -0.736185 \\ 0.032951 & 0.710370 \\ -1.188722 & 1.443067 \\ 2.058203 & -0.046474 \\ -1.263421 & 2.678123 \\ -3.184206 & 1.099470 \\ -3.796644 & -1.564264 \\ -3.639743 & -2.154314 \\ 1.468721 & -2.051315 \\ 3.089374 & -0.061512\end{array}$

$-0.204133$

$-0.033954$

0.101670

0.066490

$-0.081962$

$-0.205104$

0.035340

$-0.005228$

$-0.149956$

0.291188

$-0.218525$

$-0.334463$

0.866343

$-0.636727$

0.512442

$-0.003418$

9H-guanine $\left(\pi \pi^{*} \mathrm{Lb} / \pi \pi^{*} \mathrm{La}_{\mathrm{Cl}} \mathrm{C}-539.296605 \mathrm{au}\left({ }^{1} \mathrm{La}_{\mathrm{a}}\right)\right.$

$\begin{array}{rrrr}\mathrm{N} & -2.301836 & 0.641195 & -0.174884 \\ \mathrm{~N} & -1.176675 & -1.413269 & 0.007018 \\ \mathrm{~N} & 1.315829 & 1.174622 & 0.029627 \\ \mathrm{~N} & 1.259293 & -1.201369 & 0.028235 \\ \mathrm{~N} & -3.585590 & -1.322871 & -0.089934 \\ \mathrm{C} & -2.308876 & -0.738853 & -0.123072 \\ \mathrm{C} & -0.058535 & -0.733212 & 0.071461 \\ \mathrm{C} & 0.043783 & 0.708904 & -0.006435\end{array}$


Table S1. Cartesian coordinates $(\AA)$ of the CASSCF optimized structures obtained in the paper. 6-31G(d,p) basis set (cont).

$\begin{array}{rrr}-1.185993 & 1.426483 & -0.153835 \\ 2.053287 & -0.041517 & 0.265098 \\ -1.246127 & 2.659460 & -0.248686 \\ -3.180585 & 1.096951 & -0.288150 \\ -3.828793 & -1.641120 & 0.827257 \\ -3.646972 & -2.096240 & -0.719738 \\ 1.479921 & -2.008951 & 0.571499 \\ 3.094525 & -0.073794 & 0.004542\end{array}$

7H-guanine (gs)min: -539.512745 au

$\begin{array}{lrrr}\mathrm{C} & -0.002012 & -0.653507 & -0.005349 \\ \mathrm{C} & -0.006067 & 0.702076 & -0.012233 \\ \mathrm{C} & 2.025236 & -0.052139 & 0.002399 \\ \mathrm{C} & -1.227356 & 1.458764 & -0.001001 \\ \mathrm{C} & -2.229500 & -0.785909 & -0.000434 \\ \mathrm{O} & -1.368199 & 2.675576 & 0.011414 \\ \mathrm{~N} & 1.286501 & -1.125551 & -0.002367 \\ \mathrm{~N} & 1.308285 & 1.095718 & 0.003855 \\ \mathrm{~N} & -2.316406 & 0.593191 & -0.000412 \\ \mathrm{~N} & -1.127767 & -1.443065 & -0.012003 \\ \mathrm{~N} & -3.420108 & -1.441724 & 0.000555 \\ \mathrm{H} & -3.208160 & 1.034949 & -0.006666 \\ \mathrm{H} & -4.292001 & -0.974694 & 0.000922 \\ \mathrm{H} & -3.396955 & -2.432554 & 0.006245 \\ \mathrm{H} & 3.095815 & -0.051149 & 0.004478 \\ \mathrm{H} & 1.650423 & 2.026808 & 0.005109\end{array}$

7H-guanine ${ }^{1}\left(\text { no } \pi^{*}\right)_{\min }$ - $-539.361416 \mathrm{au}$

$\begin{array}{lrrr}\mathrm{N} & 1.706211 & -0.145986 & 0.114781 \\ \mathrm{~N} & -0.031550 & -1.745379 & 0.095763 \\ \mathrm{~N} & -1.652032 & 1.367717 & -0.214175 \\ \mathrm{~N} & -2.261800 & -0.767142 & -0.072932 \\ \mathrm{~N} & 2.162498 & -2.389504 & 0.354981 \\ \mathrm{C} & 0.871125 & 0.987292 & 0.033694 \\ \mathrm{C} & 1.195317 & -1.428611 & 0.170002 \\ \mathrm{C} & -0.903836 & -0.677756 & 0.002491 \\ \mathrm{C} & -0.500321 & 0.636671 & -0.083864 \\ \mathrm{C} & -2.674258 & 0.464545 & -0.202058 \\ \mathrm{O} & 1.409661 & 1.991258 & -0.816684 \\ \mathrm{H} & 2.584973 & -0.017661 & 0.565394 \\ \mathrm{H} & 2.988559 & -2.271145 & -0.190320 \\ \mathrm{H} & 1.784312 & -3.309525 & 0.294682 \\ \mathrm{H} & -1.734588 & 2.351235 & -0.311344 \\ \mathrm{H} & -3.695682 & 0.766518 & -0.301625 \\ & & & \end{array}$


Table S1. Cartesian coordinates $(\AA)$ of the CASSCF optimized structures obtained in the paper. 6-31G(d,p) basis set (cont).

7H-guanine ${ }^{1}\left(\pi \pi^{*} \mathrm{La}_{\mathrm{a}}\right)_{\min }:-539.312514 \mathrm{au}$

$\begin{array}{rrrr}\mathrm{N} & 1.253700 & -1.197822 & 0.058639 \\ \mathrm{~N} & 1.306960 & 1.133413 & -0.071174 \\ \mathrm{~N} & -2.339156 & 0.603157 & -0.026264 \\ \mathrm{~N} & -1.122119 & -1.414018 & 0.038219 \\ \mathrm{~N} & -3.389707 & -1.439605 & 0.009707 \\ \mathrm{C} & 0.000519 & -0.688346 & 0.056328 \\ \mathrm{C} & -0.011659 & 0.755726 & 0.038952 \\ \mathrm{C} & 2.085552 & -0.059029 & 0.111816 \\ \mathrm{C} & -1.209784 & 1.471662 & -0.047632 \\ \mathrm{C} & -2.249055 & -0.739849 & 0.006241 \\ \mathrm{O} & -1.411230 & 2.694919 & -0.103216 \\ \mathrm{H} & -3.224788 & 1.056791 & -0.033315 \\ \mathrm{H} & -4.283134 & -1.014339 & -0.041578 \\ \mathrm{H} & -3.317011 & -2.429528 & 0.028371 \\ \mathrm{H} & 3.062183 & -0.103357 & -0.336744 \\ \mathrm{H} & 1.620459 & 1.997014 & 0.311649\end{array}$

7H-guanine ${ }^{1}\left(\pi \pi^{*} \mathrm{Lb}\right)_{\min }:-539.288753$ au

$$
\begin{array}{r}
1.291550 \\
1.309102 \\
-2.333967 \\
-1.132138 \\
-3.404790 \\
-0.003173 \\
-0.025782 \\
2.013337 \\
-1.197620 \\
-2.237678 \\
-1.386110 \\
-3.217563 \\
-4.278383 \\
-3.360906 \\
3.071017 \\
1.664835
\end{array}
$$

$$
\begin{array}{r}
-1.175713 \\
1.107004 \\
0.598320 \\
-1.438398 \\
-1.433629 \\
-0.755753 \\
0.744428 \\
-0.042152 \\
1.449899 \\
-0.760901 \\
2.689197 \\
1.052022 \\
-0.982278 \\
-2.424370 \\
-0.031490 \\
2.030604
\end{array}
$$

$$
\begin{array}{r}
0.012492 \\
-0.049710 \\
-0.054079 \\
-0.025711 \\
0.046154 \\
-0.318328 \\
-0.194631 \\
0.067295 \\
-0.009610 \\
-0.012794 \\
0.116985 \\
-0.039096 \\
0.161229 \\
0.047684 \\
0.230908 \\
0.021212
\end{array}
$$

7H-guanine (gs/ $\pi \pi^{*}$ La) MECI: -539.359496 au (gs)

\begin{tabular}{lrrr}
$\mathrm{N}$ & -0.078632 & -1.637032 & -0.570554 \\
$\mathrm{~N}$ & 1.787557 & -0.580632 & 0.615582 \\
$\mathrm{~N}$ & -1.335831 & 1.316943 & 0.681384 \\
$\mathrm{~N}$ & -2.238557 & -0.586548 & -0.099259 \\
$\mathrm{~N}$ & 2.037271 & -1.333914 & -1.647888 \\
$\mathrm{C}$ & -0.852906 & -0.670202 & -0.210099 \\
$\mathrm{C}$ & -0.228307 & 0.542601 & 0.318542 \\
$\mathrm{C}$ & 0.940138 & 0.340794 & 1.209229 \\
$\mathrm{C}$ & 1.200522 & -0.981791 & -0.627439 \\
& & & \\
\hline
\end{tabular}


Table S1. Cartesian coordinates $(\AA)$ of the CASSCF optimized structures obtained in the paper. $6-31 \mathrm{G}(\mathrm{d}, \mathrm{p})$ basis set (cont).

$\begin{array}{rrr}-2.439692 & 0.572464 & 0.398110 \\ 1.141407 & 0.891529 & 2.259592 \\ 2.339670 & -1.188675 & 1.184375 \\ 2.779774 & -0.691758 & -1.819953 \\ 1.569736 & -1.643756 & -2.471675 \\ -3.419311 & 0.969590 & 0.589817 \\ -1.324025 & 2.180363 & 1.168013\end{array}$

9H-6OH-guanine ${ }^{1}(\mathrm{gs})_{\min }:-539.509041 \mathrm{au}$

$$
\begin{array}{r}
1.678015 \\
-0.068031 \\
-1.704408 \\
-2.262161 \\
2.138199 \\
1.192699 \\
-0.907645 \\
-0.568898 \\
0.802663 \\
-2.679137 \\
1.262199 \\
2.206302 \\
3.064282 \\
1.848342 \\
-2.827795 \\
-3.717067
\end{array}
$$

$$
\begin{array}{r}
-0.154293 \\
-1.785886 \\
1.380812 \\
-0.780912 \\
-2.401831 \\
-1.418829 \\
-0.747551 \\
0.583088 \\
0.848231 \\
0.534695 \\
2.088826 \\
2.058656 \\
-2.141018 \\
-3.312108 \\
-1.595989 \\
0.790740
\end{array}
$$

$$
\begin{array}{r}
0.109476 \\
0.028125 \\
-0.073238 \\
-0.086910 \\
0.203019 \\
0.096992 \\
-0.018574 \\
-0.012751 \\
0.054908 \\
-0.115927 \\
0.066851 \\
0.125584 \\
-0.042272 \\
-0.068610 \\
-0.106431 \\
-0.167831
\end{array}
$$

9H-6OH-guanine ${ }^{1}\left(\pi \pi^{*} \mathrm{La}\right)_{\min }:-539.308273 \mathrm{au}$

$$
\begin{array}{r}
1.719799 \\
-0.146017 \\
-1.645327 \\
-2.289462 \\
2.118392 \\
1.206488 \\
-0.926656 \\
-0.552048 \\
0.869666 \\
-2.675587 \\
1.257831 \\
2.201255 \\
3.071582 \\
1.833828 \\
-2.884890 \\
-3.701297
\end{array}
$$

$$
\begin{array}{r}
-0.092275 \\
-1.860641 \\
1.355274 \\
-0.804981 \\
-2.381872 \\
-1.407376 \\
-0.843275 \\
0.566574 \\
0.857512 \\
0.530460 \\
2.123394 \\
2.141604 \\
-2.154201 \\
-3.308017 \\
-1.598324 \\
0.822772
\end{array}
$$$$
0.172838
$$$$
0.087312
$$$$
-0.092959
$$$$
-0.077307
$$$$
0.174845
$$$$
-0.068316
$$$$
-0.034078
$$$$
-0.093881
$$$$
0.061286
$$$$
-0.110838
$$$$
0.088058
$$

0.157721

0.002290

$-0.053006$

$-0.060732$

$-0.160824$

9H-6OH-guanine $\left(\mathrm{gs} / \mathrm{n}_{\mathrm{N}} \pi^{*}\right)_{\mathrm{CI}}:-539.346988 \mathrm{au}(\mathrm{gs})$

$\begin{array}{lrrr}\mathrm{N} & 1.502955 & -0.114120 & 0.689603 \\ \mathrm{~N} & -0.010575 & -1.739270 & -0.019775\end{array}$


Table S1. Cartesian coordinates $(\AA)$ of the CASSCF optimized structures obtained in the paper. 6-31G(d,p) basis set (cont).

\begin{tabular}{rrrr}
\hline & -1.729072 & 1.421990 & -0.075975 \\
$\mathrm{~N}$ & -2.242741 & -0.754787 & -0.115234 \\
$\mathrm{~N}$ & 2.213083 & -2.321065 & 0.282418 \\
$\mathrm{C}$ & 0.841647 & 0.948157 & 0.003604 \\
$\mathrm{C}$ & 1.208341 & -1.396556 & 0.218980 \\
$\mathrm{C}$ & -0.897774 & -0.666691 & -0.004089 \\
$\mathrm{C}$ & -0.593629 & 0.646037 & 0.035618 \\
$\mathrm{C}$ & -2.705476 & 0.538188 & -0.148054 \\
$\mathrm{O}$ & 1.350988 & 1.223524 & -1.235450 \\
$\mathrm{H}$ & 3.135982 & -1.982113 & 0.139975 \\
$\mathrm{H}$ & 2.007027 & -3.193429 & -0.148130 \\
$\mathrm{H}$ & -3.746570 & 0.768775 & -0.229182 \\
$\mathrm{H}$ & 2.206665 & 1.614322 & -1.150181 \\
$\mathrm{H}$ & -2.776005 & -1.589163 & -0.181448
\end{tabular}

\section{H-6OH-guanine ${ }^{1}\left(\pi \pi^{*} \mathrm{Lb}\right)_{\min }:-539.317551 \mathrm{au}$}

$$
\begin{array}{r}
1.703231 \\
-0.083509 \\
-1.689781 \\
-2.294574 \\
2.131127 \\
1.205869 \\
-0.928692 \\
-0.571092 \\
0.815409 \\
-2.693800 \\
1.293273 \\
2.235734 \\
3.091295 \\
1.852134 \\
-2.882884 \\
-3.726183
\end{array}
$$

$$
\begin{array}{r}
-0.149997 \\
-1.836399 \\
1.367693 \\
-0.791223 \\
-2.394284 \\
-1.418062 \\
-0.796781 \\
0.594002 \\
0.892044 \\
0.520076 \\
2.142376 \\
2.097695 \\
-2.152148 \\
-3.335711 \\
-1.590322 \\
0.797670
\end{array}
$$

9H-6OH-guanine (gs/ $\pi \pi^{*}$ La) MECI: -539.355331 au (gs)

$\begin{array}{lr}\mathrm{N} & -0.912543 \\ \mathrm{~N} & 0.995606 \\ \mathrm{~N} & 1.354525 \\ \mathrm{~N} & 2.525002 \\ \mathrm{~N} & 2.306044 \\ \mathrm{C} & -0.363422 \\ \mathrm{C} & 0.134475 \\ \mathrm{C} & 1.351030 \\ \mathrm{C} & 0.236193 \\ \mathrm{C} & 2.305023 \\ \mathrm{O} & -0.845903 \\ \mathrm{H} & 1.510110 \\ \mathrm{H} & 2.836409 \\ \mathrm{H} & 1.613025 \\ \mathrm{H} & -0.899081 \\ \mathrm{H} & -0.623152\end{array}$

$$
\begin{array}{r}
0.292885 \\
0.019189 \\
0.858244 \\
0.434004 \\
-1.285306 \\
0.112125 \\
0.305358 \\
0.149793 \\
0.832561 \\
0.068980 \\
1.375577 \\
-1.702896 \\
-1.872071 \\
-0.047689 \\
0.026542 \\
1.749082
\end{array}
$$

0.065689
-0.051951
-0.025345
-0.114861
0.155259
0.038483
-0.095609
-0.036044
0.025582
-0.070728
0.072884
0.123092
0.100400
0.014334
-0.127686
-0.081088 
Table S1. Cartesian coordinates $(\AA)$ of the CASSCF optimized structures obtained in the paper. 6-31G(d,p) basis set (cont).

7H-6OH-guanine ${ }^{1}$ (gs)min: -539.503898 au

$\begin{array}{lrrr}\mathrm{N} & -2.785032 & 0.294725 & -0.157214 \\ \mathrm{~N} & -1.109844 & 1.989585 & -0.056807 \\ \mathrm{~N} & 1.158650 & 1.133953 & 0.102453 \\ \mathrm{~N} & 0.619768 & -1.049077 & 0.082633 \\ \mathrm{~N} & -3.347553 & 2.527553 & -0.145409 \\ \mathrm{C} & -2.351168 & 1.581510 & -0.130065 \\ \mathrm{C} & -0.207327 & 0.995614 & 0.012522 \\ \mathrm{C} & -0.553959 & -0.342763 & -0.004435 \\ \mathrm{C} & -1.912224 & -0.654625 & -0.095882 \\ \mathrm{C} & 1.602617 & -0.113183 & 0.142203 \\ \mathrm{O} & -2.299641 & -1.923087 & -0.118787 \\ \mathrm{H} & -3.245195 & -1.947859 & -0.171595 \\ \mathrm{H} & -4.207313 & 2.233941 & -0.547223 \\ \mathrm{H} & -3.047064 & 3.441576 & -0.395157 \\ \mathrm{H} & 0.728870 & -2.034619 & 0.096537 \\ \mathrm{H} & 2.635342 & -0.385192 & 0.214691\end{array}$

7H-6OH-guanine ${ }^{1}\left(\pi \pi^{*} \mathrm{La}_{\mathrm{a}}\right)_{\min }:-539.319536 \mathrm{au}$

$-2.831999$

0.280426

$-0.190282$

$-1.096077$

1.998480

$-0.083028$

1.156244

1.176140

0.097839

0.591704

$-1.044413$

0.092177

$-3.313242$

2.515179

$-0.234674$

$-2.391778$

1.536006

$-0.175088$

$-0.171125$

1.038385

0.001422

$-0.572807$

$-0.362832$

$-0.006741$

$-1.891374$

$-0.682454$

$-0.102640$

1.647723

$-0.105361$

0.155606

$-2.301351$

$-1.953251$

$-0.114848$

$-3.245153$

$-1.958909$

$-0.180200$

$-4.256463$

2.277356

$-0.427183$

$-3.003513$

3.449805

$-0.353761$

0.686493

$-2.031593$

0.113685

2.671641

$-0.384912$

0.236178

7H-6OH-guanine ${ }^{1}\left(\mathrm{n}_{\mathrm{N}} \pi *\right)_{\min }:-539.328645 \mathrm{au}$

1.680279
-0.031686
-1.642053
-2.296699
2.157374
0.892746
1.270134
-0.945408
-0.501801
-2.687482
1.364844

-0.260581
-1.680081
1.347707
-0.733675
-2.511405
0.899561
-1.474589
-0.667595
0.608094
0.489156
1.910222

$-0.086973$

0.316728

$-0.336900$

0.119384

0.336121

$-0.166234$

0.164936

0.091723

$-0.184360$

$-0.146544$

$-0.947487$ 
Table S1. Cartesian coordinates $(\AA)$ of the CASSCF optimized structures obtained in the paper. 6-31G(d,p) basis set (cont).

$\begin{array}{rrrr} & & & \\ \mathrm{H} & 3.040828 & -2.355281 & -0.094603 \\ \mathrm{H} & 1.769767 & -3.407307 & 0.139834 \\ \mathrm{H} & -1.697611 & 2.312223 & -0.561980 \\ \mathrm{H} & -3.705745 & 0.806735 & -0.218548 \\ \mathrm{H} & 2.196923 & 2.205503 & -0.613598\end{array}$

7H-6OH-guanine ${ }^{1}\left(\pi \pi^{*} \mathrm{Lb}\right)_{\min }:-539.284215 \mathrm{au}$

$\begin{array}{lrrr}\mathrm{N} & -2.822002 & 0.315298 & -0.166465 \\ \mathrm{~N} & -1.116369 & 2.031848 & -0.046535 \\ \mathrm{~N} & 1.148186 & 1.172179 & 0.109517 \\ \mathrm{~N} & 0.613292 & -1.024692 & 0.076425 \\ \mathrm{~N} & -3.367281 & 2.552496 & -0.132530 \\ \mathrm{C} & -2.382602 & 1.585636 & -0.127577 \\ \mathrm{C} & -0.199972 & 1.033903 & 0.018128 \\ \mathrm{C} & -0.531707 & -0.309031 & -0.002610 \\ \mathrm{C} & -1.878902 & -0.699671 & -0.097943 \\ \mathrm{C} & 1.634827 & -0.165975 & 0.144151 \\ \mathrm{O} & -2.270219 & -1.984518 & -0.122458 \\ \mathrm{H} & -3.215504 & -2.007338 & -0.150568 \\ \mathrm{H} & -4.224358 & 2.271929 & -0.551353 \\ \mathrm{H} & -3.038772 & 3.446255 & -0.420697 \\ \mathrm{H} & 0.670855 & -2.019724 & 0.080723 \\ \mathrm{H} & 2.659455 & -0.450544 & 0.218257\end{array}$

7H-6OH-guanine (gs/ $\pi \pi^{*}$ La)MECI: -539.349443 au (gs)

0.107799

1.546071

2.298767

1.193183

$-0.807117$

0.319443

0.259244

0.827019

1.517125

2.082709

0.020765

$-1.591294$

$-0.646358$

2.520905

$-0.284062$

1.085062

$$
\begin{array}{r}
-1.531799 \\
-0.127950 \\
1.975297 \\
1.546801 \\
-0.129537 \\
-0.764693 \\
-0.759565 \\
0.572453 \\
0.859442 \\
2.343313 \\
-1.165396 \\
-0.002286 \\
0.605204 \\
3.201453 \\
-2.062018 \\
1.504747
\end{array}
$$




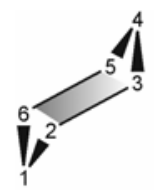

${ }^{4} \mathrm{C}_{1}$

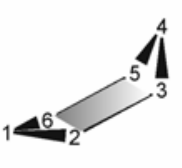

${ }^{1,4} \mathbf{B}$

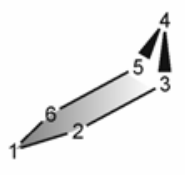

${ }^{4} \mathbf{E}$

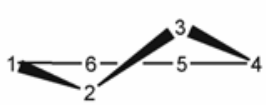

${ }^{3} \mathbf{S}_{2}$

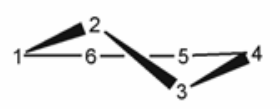

${ }^{2} \mathbf{H}_{3}$

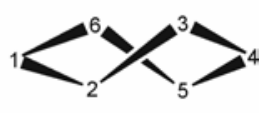

${ }^{6} \mathbf{T}_{2}$

Figure SI1. Examples of the six canonical shapes of puckered six-membered rings according to the Boeyens nomenclature: ${ }^{17,18,19} C$ (chair), B (boat), E (envelope/halfboat/sofa), $S$ (screw-boat), $H$ (half-chair), and $T$ (twisted-boat/skew-boat). IUPAC nomenclature ${ }^{20}$ tends to combine $H$ and $S$ in a single class, whereas the symbol $S$ has also been used for the $T$ conformation. ${ }^{21}$ Super- and subscripts correspond to out-ofplane atoms (up and down, respectively).

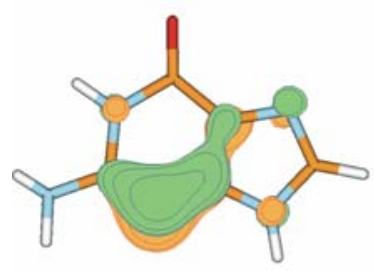

HOMO-1

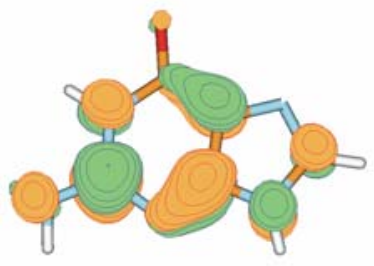

LUMO

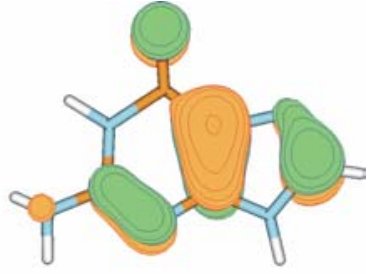

HOMO

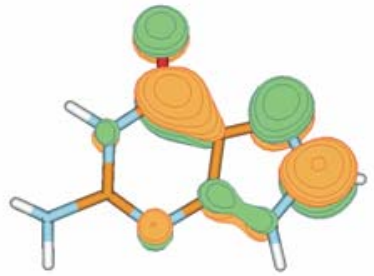

$\mathrm{LUMO}+1$

Figure SI2. The four most relevant natural orbitals in the CASSCF wave function of the 9H-guanine molecule at the FC geometry. Labeling is based on the occupation number of the corresponding natural orbital. 


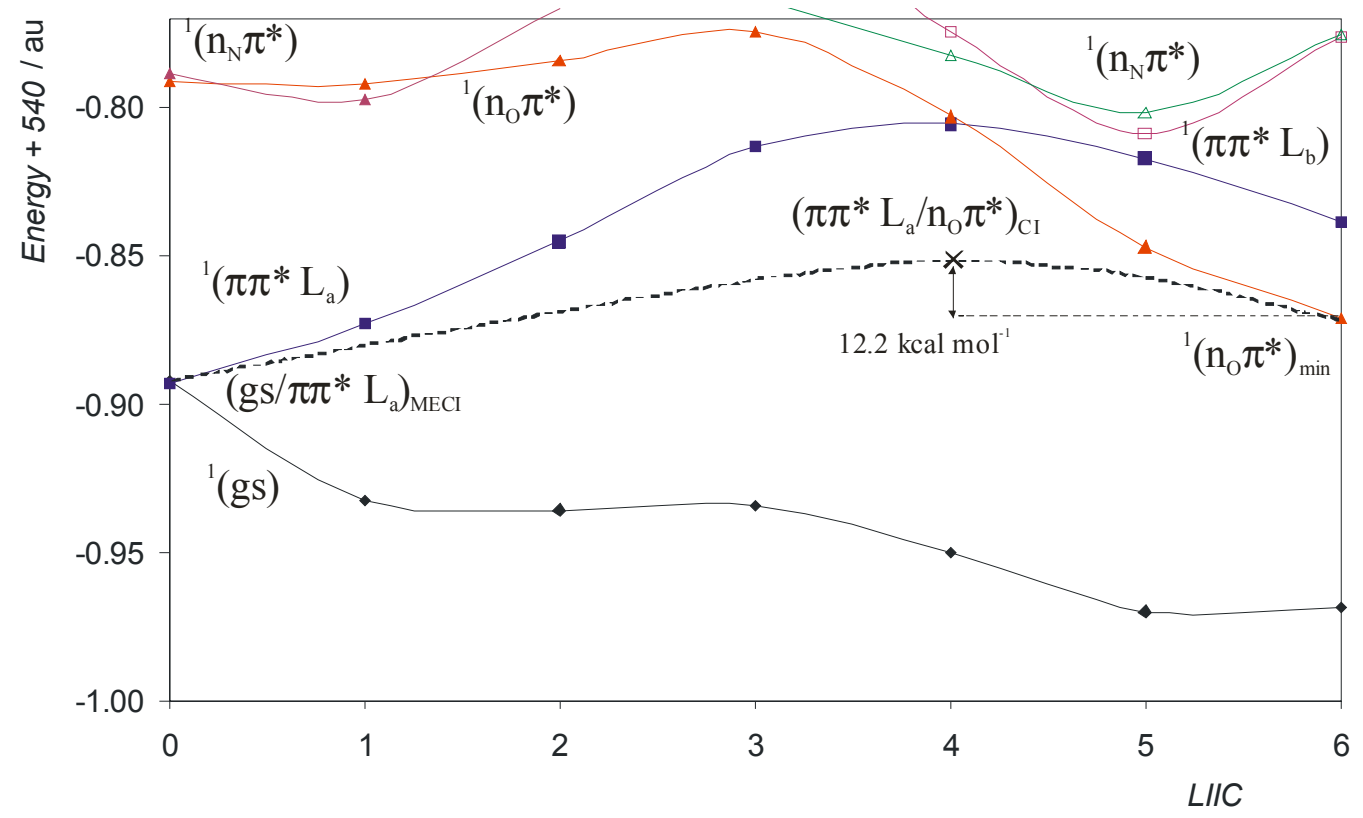

Figure SI3. Linear Interpolation in Internal Coordinates (LIIC) profile from (gs/ $\pi \pi^{*}$ $\left.L_{a}\right)_{M E C I}$ and ${ }^{1}\left(n_{O} \pi^{*}\right)_{\min }$ in $9 H$-guanine. The barrier to access the ${ }^{1}\left(\pi \pi^{*} L_{a}\right)$ state PEH (not necessarily at the MECI) from ${ }^{1}\left(n_{O} \pi^{*}\right)_{\min }$ is estimated by using the position of the $C I\left(\pi \pi^{*}\right.$ $\left.L_{a} / n_{O} \pi^{*}\right)_{C l}$, computed independently as MECP. 

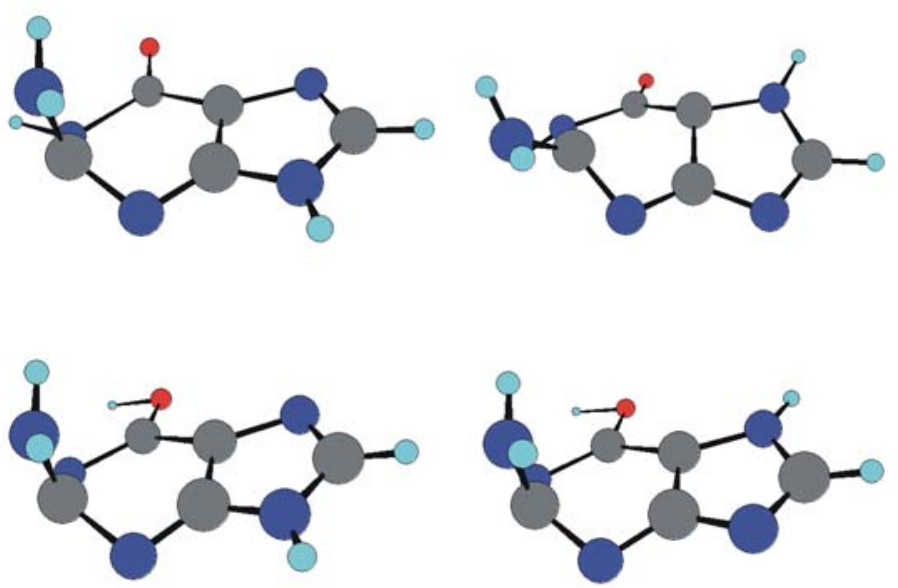

Figure SI4. Structure of the conical intersection $\left(g s / \pi \pi^{*} L_{a}\right)_{M E C I}$ in the four studied molecules. They have been obtained as Minimum Energy Conical Intersections (MECIs). These structures and those obtained as Minimum Energy Crossing Points (MECPs), that is, computed without NACMEs, were in practice identical.
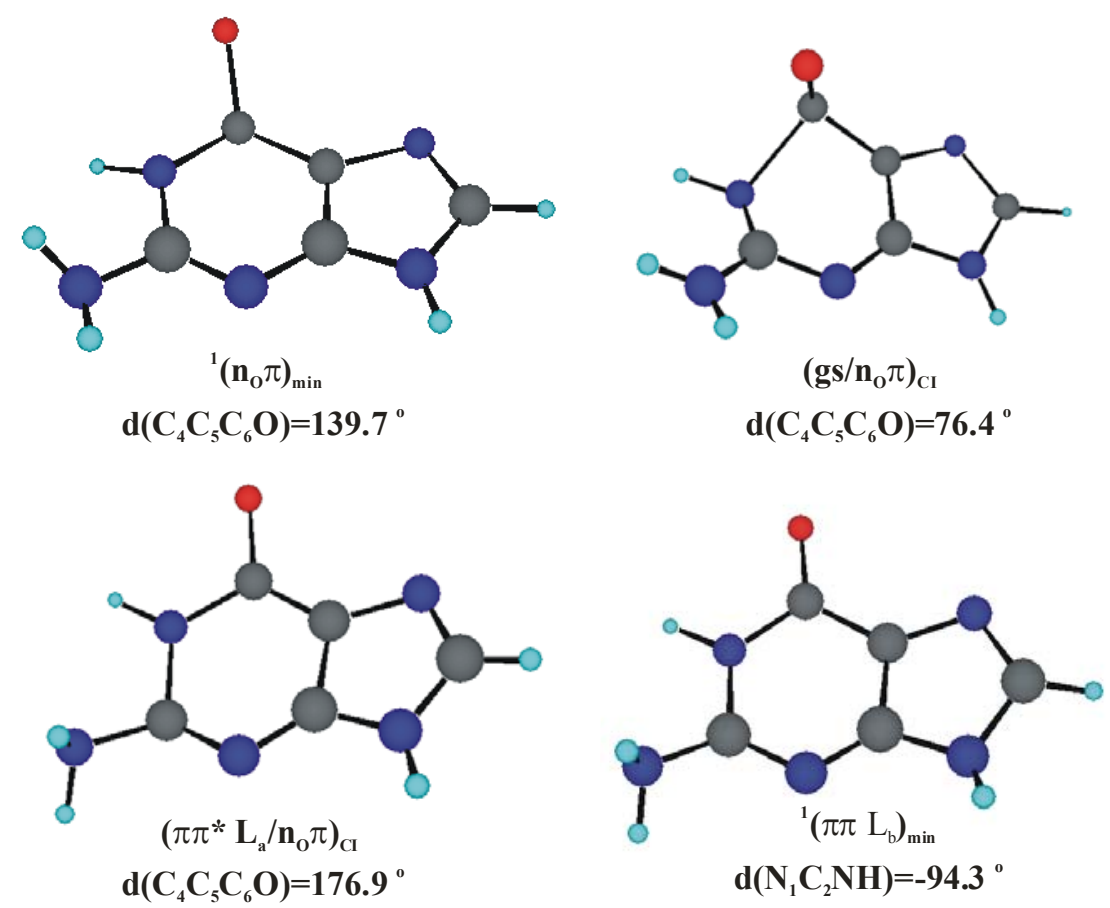

Figure SI5. Structures and most relevant coordinate of some of the critical points computed in the PEHs of the 9H-guanine molecule. 


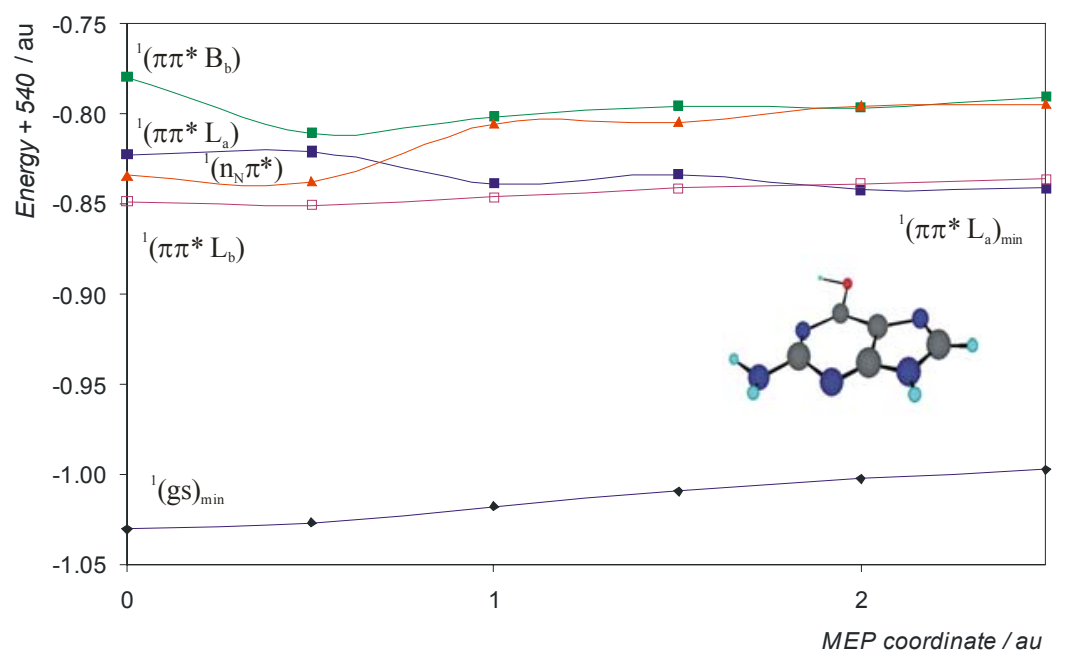

Figure SI6. Low-lying singlet excited states of $9 \mathrm{H}$-6OH-guanine computed at the CASPT2//CASSCF level from the Franck-Condon ground-state geometry along the Minimum Energy Path (MEP) on the ${ }^{1}\left(\pi \pi^{*} L_{a}\right)$ state.

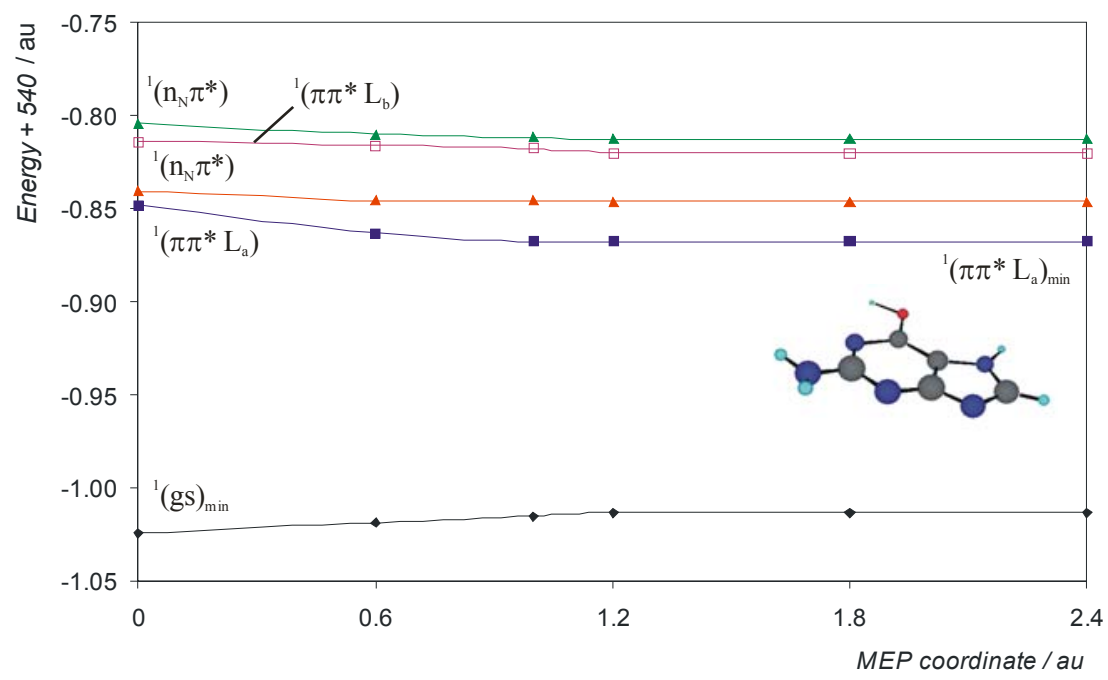

Figure SI7. Low-lying singlet excited states of 7H-6OH-guanine computed at the CASPT2//CASSCF level from the Franck-Condon ground-state geometry along the Minimum Energy Path (MEP) on the ${ }^{1}\left(\pi \pi^{*} L_{a}\right)$ state. 


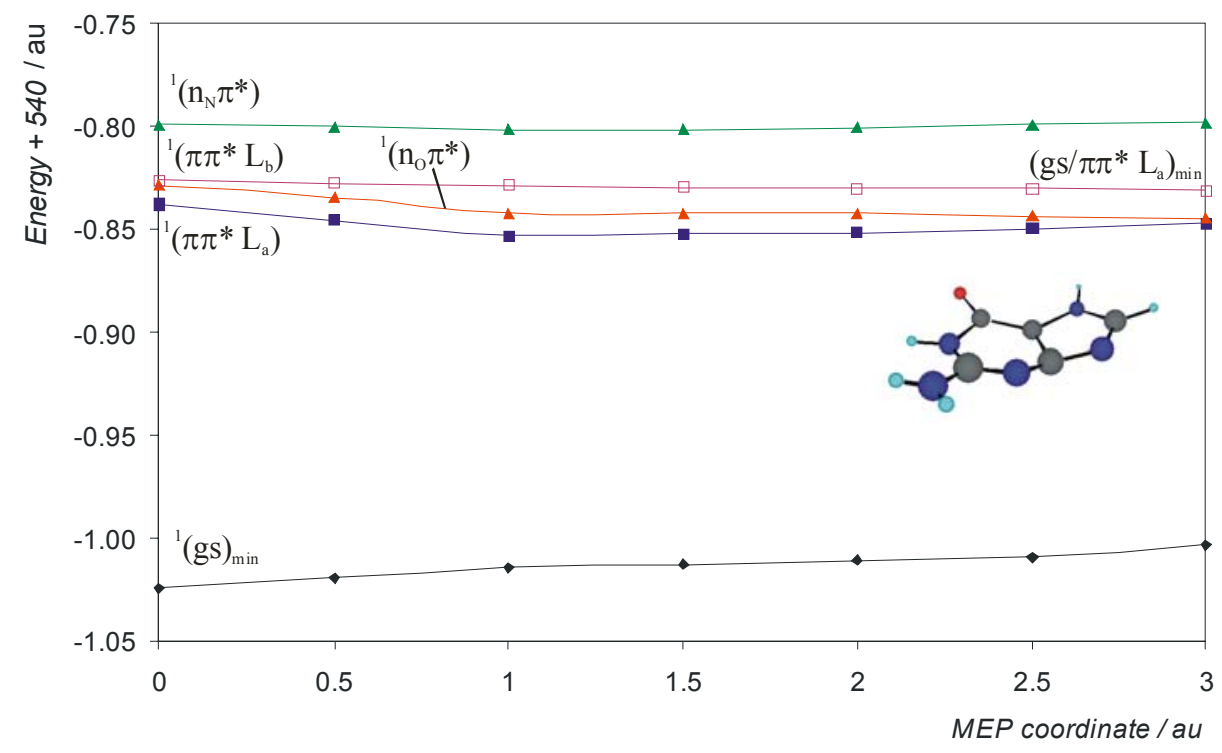

Figure SI8. Low-lying singlet excited states of 7 H-guanine computed at the CASPT2//CASSCF level from the Franck-Condon ground-state geometry along the Minimum Energy Path (MEP) on the ${ }^{1}\left(\pi \pi^{*} L_{b}\right)$ state.

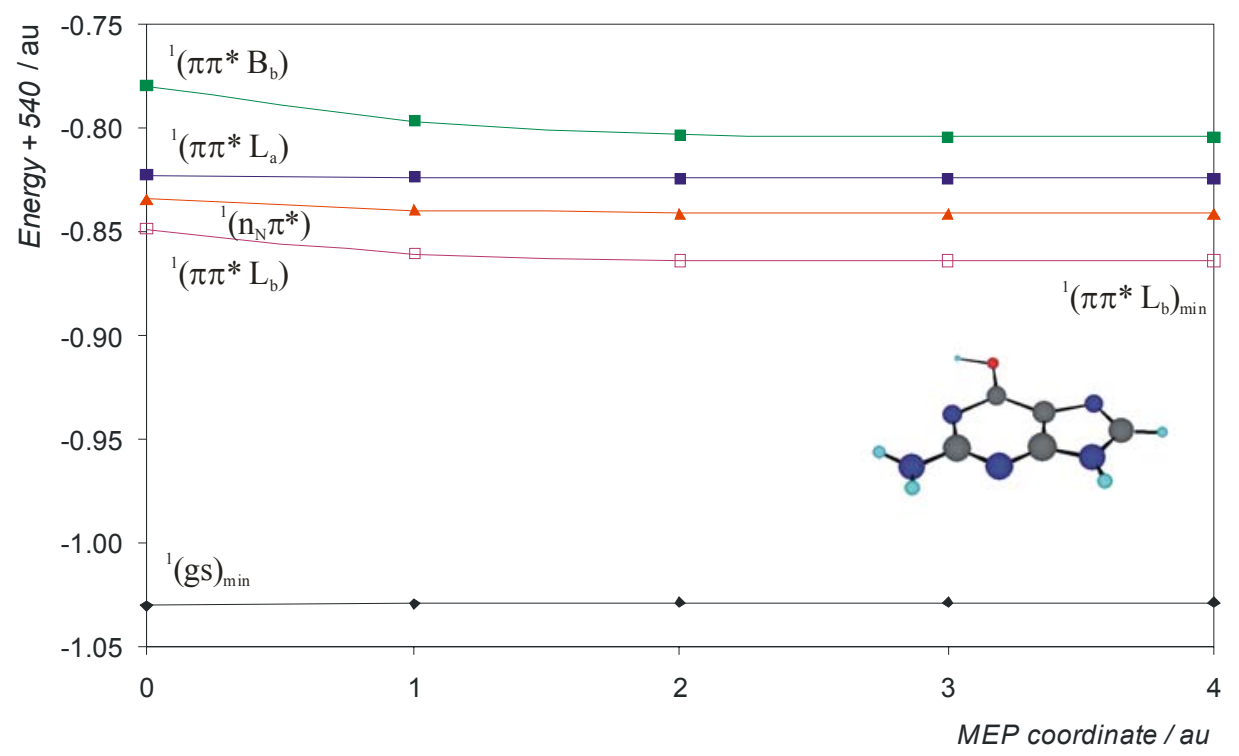

Figure SI9. Low-lying singlet excited states of 9H-6OH-guanine computed at the CASPT2//CASSCF level from the Franck-Condon ground-state geometry along the Minimum Energy Path (MEP) on the ${ }^{1}\left(\pi \pi^{*} L_{b}\right)$ state. 


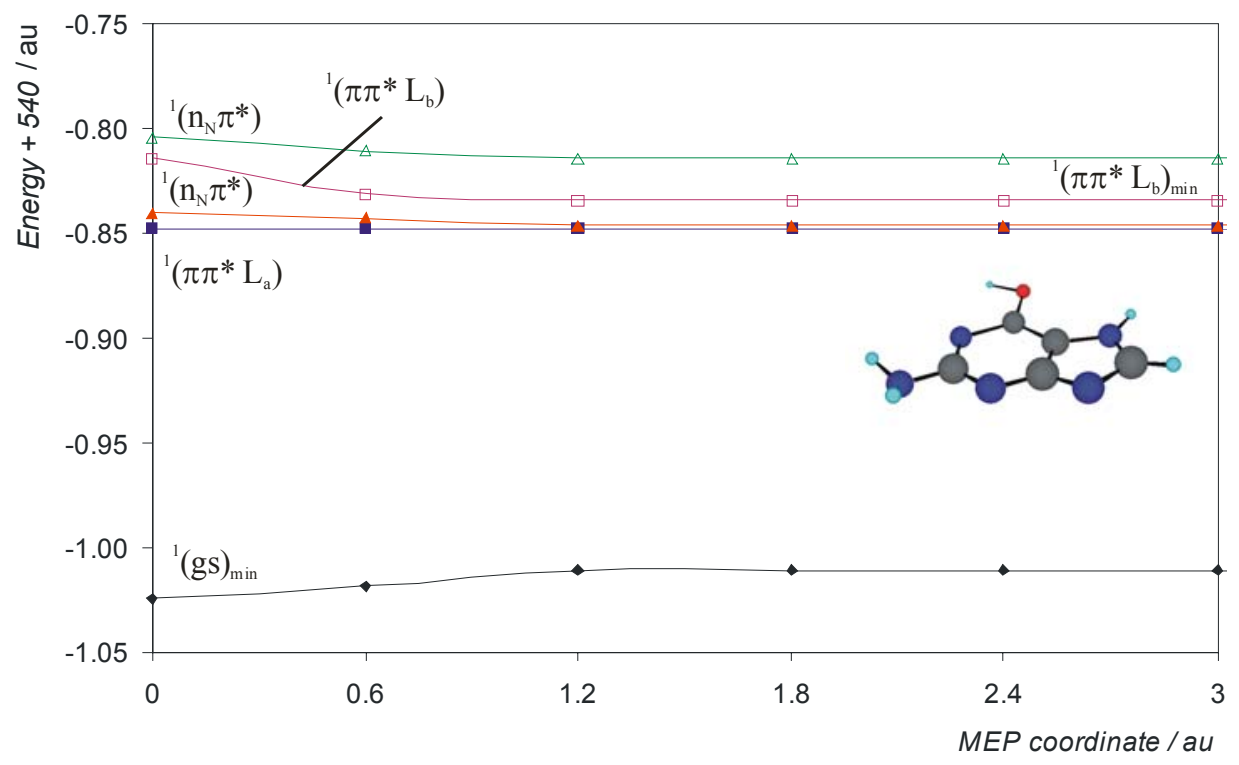

Figure SI10. Low-lying singlet excited states of 7H-6OH-guanine computed at the CASPT2//CASSCF level from the Franck-Condon ground-state geometry along the Minimum Energy Path (MEP) on the ${ }^{1}\left(\pi \pi^{*} L_{b}\right)$ state

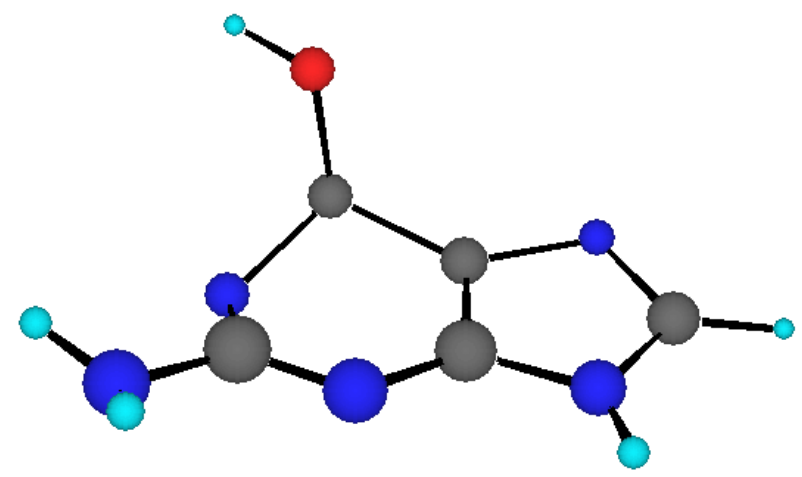

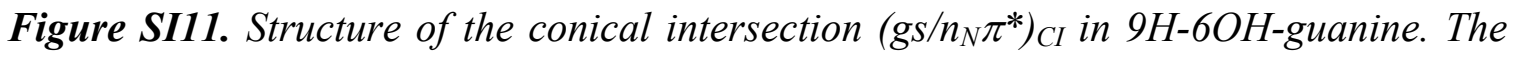
dihedral angle $d\left(C_{4} C_{5} C_{6} O\right)$ is optimized as 95.2 . 


\section{References}

(1) Andersson, K.; Malmqvist, P.-Å.; Roos, B. O. J. Chem. Phys. 1992, 96, 1218-1226.

(2) Roos, B. O.; Fülscher, M. P.; Malmqvist, P.-Å; Serrano-Andrés, L.; Pierloot, K.; Merchán, M. Adv. Chem. Phys. 1996, 93, 219-331.

(3) Merchán, M.; Serrano-Andrés, L.; Fülscher, M. P.; Roos, B. O. In: Recent Advances in Multireference Methods. Hirao, K. Ed; World Scientific Publishing, Singapore, 1999.

(4) Merchán, M.; Serrano-Andrés, L. In: Computational Photochemistry; Olivucci, M. Ed; Elsevier Publishing: Amsterdam, 2005

(5) Forsberg, J.; Malmqvist, P.-Å. Chem. Phys. Lett. 1997, 274, 196-204.

(6) Andersson, K.; Barysz, M.; Bernhardsson, A.; Blomberg, M. R. A.; Carissan, Y.; Cooper, D. L.; Cossi, M.; Fülscher, M. P.; Gagliardi, L.; de Graaf, C.; Hess, B.; Hagberg, G.; Karlström, G.; Lindh, R.; Malmqvist, P.-Å.; Nakajima, T.; Neogrády, P.; Olsen, J.; Raab, J.; Roos, B. O.; Ryde, U.; Schimmelpfennig, B.; Schütz, M.; Seijo, L.; Serrano-Andrés, L.; Siegbahn, P. E. M.; Stålring, J.; Thorsteinsson, T.; Veryazov, V.; Widmark, P.-O. MOLCAS, version 6.0; Department of Theoretical Chemistry, Chemical Centre, University of Lund: Lund, Sweden, 2004.

(7) Frisch, M. J.; Trucks, G. W.; Schlegel, H. B.; Scuseria, G. E.; Robb, M. A.; Cheeseman, J. R.; Montgomery, J. A., Jr.; Vreven, T.; Kudin, K. N.; Burant, J. C.; Millam, J. M.; Iyengar, S. S.; Tomasi, J.; Barone, V.; Mennucci, B.; Cossi, M.; Scalmani, G.; Rega, N.; Petersson, G. A.; Nakatsuji, H.; Hada, M.; Ehara, M.; Toyota, K.; Fukuda, R.; Hasegawa, J.; Ishida, M.; Nakajima, T.; Honda, Y.; Kitao, O.; Nakai, H.; Klene, M.; Li, X.; Knox, J. E.; Hratchian, H. P.; Cross, J. B.; Bakken, V.; Adamo, C.; Jaramillo, J.; Gomperts, R.; Stratmann, R. E.; Yazyev, O.; Austin, A. J.; Cammi, R.; Pomelli, C.; Ochterski, J. W.; Ayala, P. Y.; Morokuma, K.; Voth, G. A.; Salvador, P.; Dannenberg, J. J.; Zakrzewski, V. G.; Dapprich, S.; Daniels, A. D.; Strain, M. C.; Farkas, O.; Malick, D. K.; Rabuck, A. D.; Raghavachari, K.; Foresman, J. B.; Ortiz, J. V.; Cui, Q.; Baboul, A. G.; Clifford, S.; Cioslowski, J.; Stefanov, B. B.; Liu, G.; Liashenko, A.; Piskorz, P.; Komaromi, I.; Martin, R. L.; Fox, D. J.; Keith, T.; Al-Laham, M. A.; Peng, C. Y.; Nanayakkara, A.; Challacombe, M.; Gill, P. M. W.; Johnson, B.; Chen, W.; Wong, M. W.; Gonzalez, C.; Pople, J. A. Gaussian 03, Inc.: Wallingford CT, 2004.

(8) De Vico, L.; Olivucci, M.; Lindh, R. J. Chem. Theory Comp. 2005, 1, 1029-1037.

(9) Anglada, J. M.; Bofill, J.M. J. Comput. Chem. 1997, 18, 992-1003.

(10) Müller, K.; Brown, L. D. Theor. Chim. Acta. 1979, 53, 75-93.

(11) Andersen, H. C. J. Comp. Phys. 1983, 52, 24-34.

(12) Stålring, J.; Bernhardsson, A.; Lindh, R. Mol. Phys. 2001, 99, 103-114.

(13) Groenhof, G.; Bouxin-Cademartory, M.; Hess, B.; De Visser, S. P.; Berendsen, H. J. C.; Olivucci, M.; Mark, A. E.; Robb, M. A. J. Am. Chem. Soc. 2004, 126, 4228-4232.

(14) Weingart, O.; Schapiro, I.; Buss, V. J. Phys. Chem. B 2007, 111, 3782-3788.

(15) Tully, J. C. J. Chem. Phys. 1990, 93, 1061-1071.

(16) Doltsinis, N. L.; Marx, D. J. Theor. Comp. Chem. 2002, 1, 319-349.

(17) Barbatti, M.; Lishcka, H. J. Phys. Chem. A 2007, 111, 2852-2858.

(18) Cremer D.; Pople, J. A. J. Am. Chem. Soc. 1975, 97, 1354-1358.

(19) Boeyens, J. C. A. J. Cryst. Mol. Struct. 1978, 8, 317-320.

(20) Hill, A. D.; Reilly, P. J. J. Chem. Inf. Model. 2007, 47, 1031-1035.

(21) Bérces, A.; Whitfield, D. M.; Nukada, T. Tetrahedron 2001, 57, 477-491. 